Research Journal of Medical Sciences 6 (4): 170-174, 2012

ISSN: $1815-9346$

(C) Medwell Journals, 2012

\title{
Is Rotavirus Infection Still Responsible for Acute Gastroenteritis and Severe Diarrhea among Child ren in Holy Makkah?
}

\author{
Ahmad Mohammad Ashshi \\ Department of Laboratory Medicine, Faculty of Applied Medical Sciences, \\ Umm-Al Qura University, Holy Makkah, Saudi Arabia
}

\begin{abstract}
Acute gastroenteritis and severe diarrhea is a leading cause of preventable death in infants and young children worldwide. It ranks second to neonatal deaths as the major cause of childhood mortality. In this regard, rotavirus infection is the most important microbial causative agent, particularly in developing countries. Coherently, this study was designed to assess the prevalence of rotavirus infection among young children with acute gastroenteritis and severe diarrhea in Holy Makkah, Saudi Arabia and evaluate the specificity of Latex Agglutination Test (LAT) in detection of rotavirus infection in comparison with Reverse-Transcription Polymerase Chain Reaction (RT-PCR). During the period from March to September 2011, stool samples were collected from 100 children (of both sex and under 5 years of age) with acute gastroenteritis and severe diarrhea and admitted to the pediatric wards at the Garwal Hospital in Holly Makkah. For detection of rotavirus infection, the samples were first examined by LAT and then by RT-PCR using specific primer sequences for human rotavirus $V P 4$ and $V P 7$ genes. Sixteen samples of these 100 samples (16\%) were positive for rotavirus by LAT. However, the genetic materials of rotavirus ( $V P 4$ and $V P 7$ genes) were only detected in 4 samples of these 100 samples $(4 \%)$ by RT-PCR amplification. The samples that tested negative with LAT were also negative with RT-PCR. Overall, this study indicated that rotavirus infection still responsible for acute gastroenteritis and severe diarrhea among children in Holy Makkah with a prevalence of $4 \%$. In turn, this may help in assessing the success of the rotavirus vaccine in the future. In addition, this study reflects the low specificity of LAT than RT-PCR for detection of rotavirus infection.
\end{abstract}

Key words: Rotavirus, infection, children, Latex Agglutination test, RT-PCR, Saudi Arabia

\section{INTRODUCTION}

Diarrheal disease remains a major cause of childhood mortality, annually accounting for $>1.8$ million deaths among children $<5$ years of age (Boschi-Pinto et al., 2007). Of the $>50$ pathogens capable of causing gastroenteritis, rotavirus is the most important cause of severe, dehydrating gastroenteritis in infants and young children worldwide (Glass et al., 2006; Wang et al., 2010). Mostly, all children experience rotavirus infection by the age 5 years (Parashar et al., 2003) and an estimated 527,000 rotavirus-associated deaths occur in developing countries (WHO, 2011, 2009; Steele et al., 2009). Therefore, control of rotavirus infection would potentially not only lead to a significant reduction of fatalities in developing countries but also, considerable healthcare cost savings (Widdowson et al., 2009; Clark et al., 2009). Presently, two live and orally-administrable rotavirus vaccines are currently licensed in many countries after they had gone through large scale safety and efficacy trials (RuizPalacios et al., 2006; Vesikari et al., 2006; Wang et al., 2010).
Rotaviruses are triple-layered icosahedral particles and their genomes consist of 11 segments of doublestranded RNA. Based on epitopes on the inner capsid, rotaviruses are subdivided into 7 groups (A-G) but most human infections are due to Group A rotaviruses. Rotaviruses are further classified according to the genetic and antigenic diversity of the 2 outer capsid proteins, VP4 (P serotype) and VP7 (G serotype) (Cunliffe and Nakagomi, 2005). In addition as $\mathrm{G}$ and $\mathrm{P}$ type-specific immunity is believed to play a role in protection against disease, the epidemiology of $\mathrm{G}$ and $\mathrm{P}$ serotypes (and genotypes) of circulating strains, forms a critical knowledge base for the development and implementation of rotavirus vaccines (Kapikian and Hoshino, 2007). To date, $19 \mathrm{G}$ serotypes and $27 \mathrm{P}$ types have been defined. However, only a few P- and G- and P/G combinations have been found in human (Kheyami et al., 2006, 2008a, b; Chandran et al., 2010). Because the clinical manifestations of rotavirus gastroenteritis are not distinct enough to permit a specific diagnosis, specimens must be examined in the laboratory. Several techniques were developed for detection of rotavirus infection such as Poliacrylamide Gel 
Electrophoresis (PAGE), Immunofluorescence (IF), Radio Immunoassay (RIA) and Enzyme-linked Immunosorbrnt Assay (ELISA), Reverse Passive Hemagglutination (RPH), Latex Agglutination Test (LAT), electron microscopic examination and more recently, a Reverse Transcription Polymerase Chain Reaction (RT-PCR) (Fernandes et al., 2000; Buser et al., 2001; Marshall et al., 2003; Muller and Johne, 2007).

With respect to Saudi Arabia, some recent studies indicated that the proportion of active gastroenteritis cases in Saudi Arabia that were rotavirus positive range from $32-46 \%$ among hospitalized children with variation by location, month and season (Kheyami et al., 2006, $2008 \mathrm{a}, \mathrm{b}$; Nabi et al., 2008). Indeed, there is a paucity of data on the prevalence of rotavirus infection in Holy Makkah. Therefore, this study was designed to estimate the prevalence of rotavirus infection among children in Holy Makkah to inform the need for a rotavirus immunization program and evaluate the specificity of latex test in detection of rotavirus infection in comparison with RT-PCR amplification technique.

\section{MATERIALS AND METHODS}

Patients and sampling: During the period from March to September, 2011 stool samples were randomly obtained from 100 children (of both sex and age $<5$ years) who were admitted to Garwal Hospital of Obstetrics and Gynecology, Holy Makkah with acute gastroenteritis and sever non-bloody diarrhea. Diarrhea was defined as the passage of 3 or more looses than normal stools within a $24 \mathrm{~h}$ period (as described by care giver/mother on presentation at clinic). Each collected sample was rapidly separated into 2 sterile tubes: one contained $1 \%$ glycerol (to keep the integrity of the genetic materials of the sample) and the second without glycerol (Fig. 1). The samples without glycerol were employed for Latex Agglutination Test (LAT) for detection of rotavirus antigens while those with glycerol were stored at $-80^{\circ} \mathrm{C}$

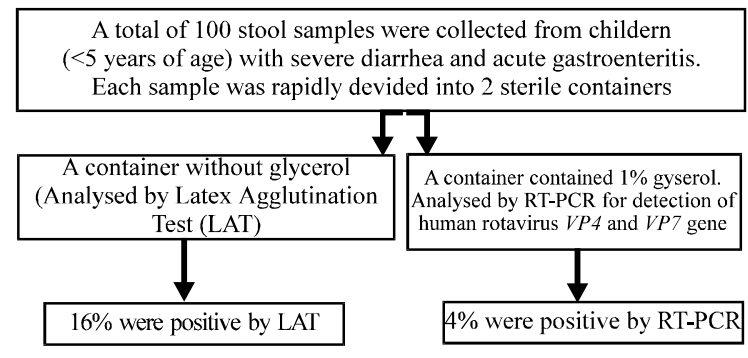

Fig. 1: A schematic diagram of the methodology and the results until used for RT-PCR detection of rotavirus nucleic acids in the LAT positive samples. The protocol of this study was approved by the Ethical Committee of Faculty of Applied Medical Sciences, Umm Al-Qura University and a specific questioner including the clinical and demographic data of each participant was obtained.

Detection of rotavirus by Latex Agglutination test: The fecal samples were examined by VIROTECT ROTA Rapid Latex Slid test (Ref. \#: OD038; Omega Diagnostic Ltd. UK) according to manufacturer's instructions. Briefly, each specimen was centrifuged at $1000 \times \mathrm{g}$ for $10 \mathrm{~min}$ and then $50 \mu \mathrm{L}$ of its supernatant were transferred onto each of two wells (test and control) of the test slide. Next, a drop of LAT suspension reagent and control reagent was added to the test well and the control well, respectively. Finally, the contents of each well were thoroughly mixed and the whole slide was gently and evenly rocked and rotated for 2 min. As recommended by the kit's manufacturer, a sample was considered positive for rotavirus when agglutination was clearly observed within 2 min reaction.

Detection of rotavirus by RT-PCR analysis: The stool samples that showed positivity or negativity to LAT were further examined for detection of rotavirus genetic materials by Reverse-transcription Polymerase Chain Reaction (RT-PCR) amplification technique using specific primer sequences for human rotavirus VP4 and VP7 genes. Total RNA was extracted from the samples by using the commercial RNA extraction kit (Q1A amp viral RNA mini; UK) following kit's description. Next, RT-PCR amplification processes were employed by using Qiagen One Step RT-PCR Kit (UK) and specific primers of human rotavirus VP7 and VP4 genes. The specific sequencing of VP7 sense (forward) primer was 5' GGCTTT AAA AGA GAG AATTTCCGTCTG G 3 ' and that of VP7 anti-sense (reverse) primer was 5' GGTCACATC ATA CAATTCTAATCTAAG 3 ' with a suspected PCR product size of 1062 bp while VP4 sense (forward) primer sequencing was 5 ' TGGCTTCGC CAT TT ATA GAC A 3 ' and VP4 anti-sense (reverse) primer sequencing was $5^{\prime}$ ATT TCGGAC CAT TTATAA CC 3 ' with a suspected PCR product size of $876 \mathrm{bp}$. The RT-PCR amplification reactions for either VP7 or VP4 gene was performed at $50^{\circ} \mathrm{C}$ for $30 \mathrm{~min}, 95^{\circ} \mathrm{C}$ for $15 \mathrm{~min}$ followed by 35 cycles of $94^{\circ} \mathrm{C}$ for $1 \mathrm{~min}, 60^{\circ} \mathrm{C}$ for $1 \mathrm{~min}, 72^{\circ} \mathrm{C}$ for $1 \mathrm{~min}$ and a final extension at $72^{\circ} \mathrm{C}$ for $10 \mathrm{~min}$ and then hold at $4^{\circ} \mathrm{C}$. Finally, all amplified PCR products were examined by gel electrophoresis in $2 \%$ agarose gels containing $4 \mu \mathrm{g}$ of ethidium bromide $/ \mathrm{mL}$ under standard conditions. 


\section{RESULTS AND DISCUSSION}

As shown in Fig. 1 out of the total 100 samples, 16 samples $(16 \%)$ were LAT positive and this means that there is a shedding of rotavirus antigenic structures in their stools. On the other hand, it has previously been reported that LAT is a simple and fast assay however, its specificity may vary according to the commercial kit used. Therefore, these total 100 samples including those showed LAT positivity were further analyzed by RT-PCR to confirm the presence of the genetic materials of the human rotavirus. RT-PCR technique was employed to identify the genes of human rotavirus structural protein 4 and 7 (VP4 and VP7), respectively. As shown in Fig. 1 and 2 , the RT-PCR products indicated that both VP4 and VP7 genes were isolated only from 4 samples of these 100 samples. Moreover, samples that tested negative with latex test were also negative with RT-PCR.

Despite vaccination programs, rotavirus infection is still one of the most important causative agents of severe, dehydrating gastroenteritis and diarrhea in infants and young children worldwide including Saudi Arabia (Johargy et al., 2010; Obeid, 2011). Indeed, there is a paucity of data on its prevalence among the infant and young children in Holy Makkah. Therefore, this study was designed to highlight the disease burden and its
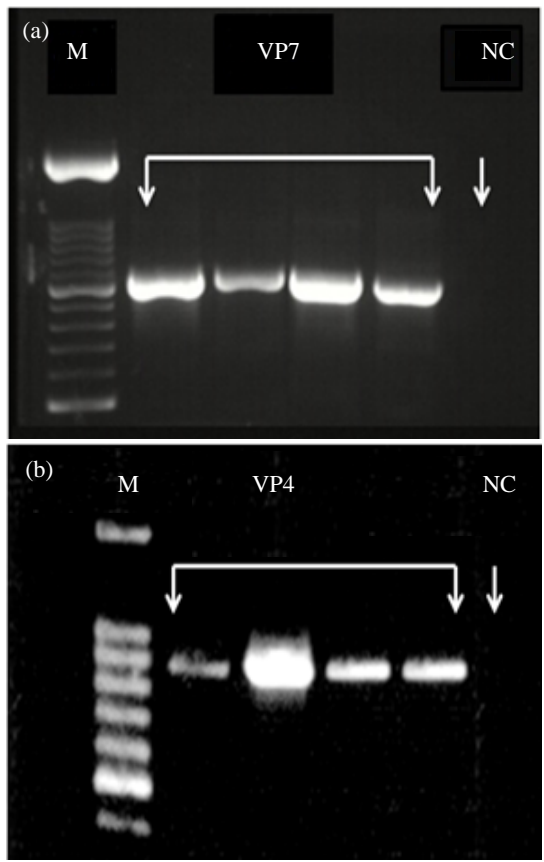

Fig. 2: A representative set of RT-PCR amplification products of human rotavirus VP7 and VP4 genes in the examined samples (M: DNA Ladder marker; NC: Negative Control) prevalence among children in Holy Makkah. A total of 100 stool samples were collected from children $<5$ years of age with acute gastroenteritis and severe diarrhea. The samples were first examined by Latex Agglutination Test (LAT) and then by RT-PCR. The prevalence of rotavirus infection among the investigated children was $4 \%$ based on RT-PCR. The LAT was less specific than RT-PCR for detection of rotavirus infection whereas it showed $16 \%$ positivity in these tested samples.

Diarrheal disease remains a major cause of childhood mortality that annually accounting for $>1.8$ million deaths among children $<5$ years of age (Boschi-Pinto et al., 2007). of the $>50$ pathogens capable of causing gastroenteritis, rotavirus is the most important one to cause severe gastroenteritis and diarrhea in infants and young children worldwide (Glass et al., 2005, 2006). Mostly, all children experience rotavirus infection by the age 5 years (Parashar et al., 2003) and an estimated 527,000 rotavirusassociated deaths occur in developing countries (WHO, 2009, 2011; Steele et al., 2009). In consistency with the findings, Johargy et al. (2010) have recently identified the frequency of viral, bacterial and parasitic enteropathogens among young children with acute diarrhoea in Saudi Arabia. In this study, the researchers specified that most of the diarrheal diseases in young children in Saudi Arabia are caused by viral etiology, particularly infection with rotavirus followed by adenoviral and astroviral infections. Over the past decades, several techniques have been developed for rotavirus diagnosis including Latex Agglutination Test (LAT), Immunofluorescence (IF), Radio Immunoassay (RIA), Reverse Passive Hemagglutination (RPH), Immunoenzimatic Assay (IEA) and Enzyme-Linked Immunosorbent Assay (ELISA), Poliacrylamide Gel Electrophoresis (PAGE), Electronic Microscopy (EM) and more recently, a Reverse Transcription Polymerase Chain Reaction (RT-PCR).

Among these assays, Latex Agglutination Test (LAT) has been reported as a simple and fast assay as required for rapid diagnosis and illness control on hospital level (Fernandes et al., 2000; Buser et al., 2001; Muller and Johne, 2007). Therefore, the collected samples were first examined by a Latex Agglutination test and their results showed $16 \%$ of rotavirus positivity. Previously, Ghazi et al. (2005) have conducted a similar study in Holy Makkah in which LAT has revealed the presence of $10 \%$ prevalence of rotavirus infection among 450 examined infants and children with gastroenteritis and diarrhea. Based on LAT results and in comparison with Ghazi's findings, the present study can estimate that the frequency of rotavirus infection in Holy Makkah is at an increase. On the other hand, other studies have been conducted in different regions of Saudi Arabia and their 
data estimated that the proportion of active rotavirus infection-associated gastroenteritis range from $32-46 \%$ among hospitalized children with variation by location, month and season (Kheyami et al., 2006, 2008a, b; Nabi et al., 2008). Moreover, in a more recent study by Obeid (2011), the rotavirus infection was assessed in 156 children with acute gastroenteritis in the Children and Maternity Hospital in Dammam by using the novel rotavirus STAT-PAK Immunochromatographical test. His results indicated that, 37 samples were shown to be positive for rotavirus $(23.7 \%)$. These studies have collectively indicated that there is a significant location and seasonal variation in rotavirus infection within Saudi Arabia with increased infection through Winter in some cities and during Summer in others. In support, many observational studies of human rotavirus disease have suggested that lower temperature, lower relative humidity and higher levels of rain are associated with increased risks of rotavirus infections (Atchison et al., 2010). Taken together, researchers support the hypothesis that the geographical position of Holy Makkah very hot and dry summer, mild winter and little rain during the year may be related to low rate of rotavirus infection if compared with other regions in Saudi Arabia.

Although, LAT has been reported as a simple and fast assay for rotavirus infections on hospital level, its sensitivity and specificity may vary (Gentsch et al., 2005; Muller and Johne, 2007). Therefore, in the present study researchers further analyzed the samples by using RT-PCR amplification technique to evaluate the sensitivity and specificity of the employed LAT for detection of rotavirus infection. On the molecular biological bases, rotaviruses possess a genome that consists of 11 segmented double-stranded $R N A$ genes that code for both structural and non-structural proteins. The outer capsid of the rotavirus is made up of two structural proteins, VP7 and VP4 which elicit neutralizing antibodies and contain independent protective antigens (Zuccotti et al., 2010; Esona et al., 2010). These proteins, encoded by specific genes of the rotavirus genome can segregate independently by the mechanism of reassortment and form the basis of the dual classification system of rotaviruses. After the virus is taken into the cell by receptor mediated endocytosis, VP7 and VP4 spikes puncture the resultant endosome resulting in a decrease in calcium ion concentration. This decrease triggers the VP7 trimer to break up into its three subunits thereby detaching from the virus. This detachment allows the virus to proceed in its invasion mechanism. Moreover, there is much hope for the use of VP7 and VP4 in vaccine development (Aoki et al., 2009; Settembre, 2011). Based on these facts, the RT-PCR amplification processes in this study were performed by using specific primer sequences of both VP4 and VP7 genes. Interestingly, only 4 of these 100 samples were positive by RT-PCR. This in turn, reflects the low specificity accuracy of LAT than RT-PCR for detection of rotavirus infection.

\section{CONCLUSION}

This study can indicate that rotavirus infection still represent an important causative agent of severe gastroenteritis among infants and young children in Holy Makkah. Finally, although LAT is a simple and fast assay for detection of rotavirus infections on hospital level, it has sub-optimal specificity than RT-PCR test.

\section{ACKNOWLEDGEMENTS}

The researcher is grateful to Dr. Adel El-Shemi and Miss Sumayah Al-Asmari (Department of Laboratory Medicine, Faculty of Applied Medical Sciences, Umm Al-Qura University) and to Dr. Sami Melebary (the pediatric wards at the Garwal Hospital in Holy Makkah) for their great help and excellent assistance.

\section{REFERENCES}

Aoki, S.T., E.C. Settembre, S.D. Trask, H.B. Greenberg, S.C. Harrison and P.R. Dormitzer, 2009. Structure of rotavirus outer-layer protein VP7 bound with a neutralizing Fab. Science, 324: 1444-1447.

Atchison, C.J., C.C. Tam, S. Hajat, W. van Pelt, J.M. Cowden and B.A. Lopman, 2010. Temperaturedependent transmission of rotavirus in Great Britain and The Netherlands. Proc. Biol. Sci., 277: 933-942.

Boschi-Pinto, C., L. Velebit and K. Shibuya, 2007. Estimating child mortality due to diarrhea in developing countries: A meta-analysis review. Bull. World Health Organ., 51: 505-516.

Buser, J., L. Risch, T. Rutz, S. Manang and J. Munzinger, 2001. Comparison of a rotavirus latex agglutination test with two rapid immunochromatographic test devices for detection of rotavirus in human feces. Eur. J. Clin. Microbiol. Infect. Dis., 20: 295-296.

Chandran, A., S. Fitzwater, A. Zhen and M. Santosham, 2010. Prevention of rotavirus gastroenteritis in infants and children: Rotavirus vaccine safety, efficacy and potential impact of vaccines. Biologics, 4: 213-229.

Clark, H.F., D. Lawley, L.A. Mallette, M.J. DiNubile and R.L. Hodinka, 2009. Decline in cases of rotavirus gastroenteritis presenting to the children's hospital of Philadelphia after introduction of a pentavalent rotavirus vaccine. Clin. Vaccine Immunol., 16: $382-386$. 
Cunliffe, N.A. and O. Nakagomi, 2005. A critical time for rotavirus vaccines: A review. Expert Rev. Vaccines, 4: $521-532$.

Esona, M.D., S. Mijatovic-Rustempasic, C. Conrardy, S. Tong and I.V. Kuzmin et al., 2010. Reassortant group A rotavirus from straw-colored fruit bat (Eidolon helvum). Emerg. Infect. Dis., Vol. 16.

Fernandes, J.V., S.M. Fonseca, J.C. Azevedo, S.M. Hde, M.H. Fonseca, M.T. Dantas and V.M. Rde, 2000. Rotavirus detection in feces of children with acute diarrhea. J. Ped., 76: 300-304.

Gentsch, J.R., A.R. Laird, B. Bielfelt, D.D. Griffin and K. Banyai et al., 2005. Serotype diversity and reassortment between human and animal rotavirus strains: Implications for rotavirus vaccine programs. J. Infect. Dis., 192: S146-S159.

Ghazi, H.O., M.A. Khan, A.M. Telmesani, B. Idress and M.F. Mahomed, 2005. Rotavirus infection in infants and young children in Makkah, Saudi Arabia. J. Pak. Med. Assoc., 55: 231-234.

Glass, R.I., J.S. Bresee, R. Turcios, T.K. Fischer, U.D. Parashar and A.D. Steele, 2005. Rotavirus vaccines: Targeting the developing world. J. Infect. Dis., 192: S160-S166.

Glass, R.I., U.D. Parashar, J.S. Bresee, R. Turcios and T.K. Fisher et al., 2006. Rotavirus vaccines: Current prospects and future challenges. Lancet., 368: 323-332.

Johargy, A., H. Ghazi and A. Mumenah, 2010. Frequency of viral, bacterial and parasitic enteropathogens among young children with acute diarrhea in Saudi Arabia. J. Pak. Med. Assoc., 60: 456-459.

Kapikian A.Z. and Y. Hoshino, 2007. Rotaviruses: To serotype or not to serotype: That is still the question. J. Infect. Dis., 195: 611-614.

Kheyami, A.M., M.Y. Areeshi, W. Dove, O. Nakagomi, N.A. Cunliffe and H.C. Anthony, 2008a. Characterization of rotavirus strains detected among children and adults with acute gastroenteritis in Gizan, Saudi Arabia. Saudi Med. J., 29: 90-93.

Kheyami, A.M., N.A. Cunliffe and C.A. Hart, 2006. Rotavirus infection in Saudi Arabia. Ann. Saudi Med., 26: 184-191.

Kheyami, A.M., T. Nakagomi, O. Nakagomi, W. Dove, C.A. Hart and N.A. Cunliffe, 2008b. Molecular epidemiology of rotavirus diarrhea among children in Saudi Arabia: First detection of G9 and G12 strains. J. Clin. Microbiol., 46: 1185-1191.

Marshall, J., J. Botes, G. Gorrie, C. Boardman and J. Gregory et al., 2003. Rotavirus detection and characterization in outbreaks of gastroenteritis in aged-care facilities. J. Clin. Virol., 28: 331-340.
Muller, H. and R. Johne, 2007. Rotaviruses diversity and zoonotic potential: A brief review. Berl. Munch. Tierarztl. Wochenschr., 120: 108-112.

Nabi, G., A.M. Kheyami and N.A. Cunliffe, 2008. Characterization of rotavirus strains detected among children and adults with acute gastroenteritis in Gizan, Saudi Arabia. Saudi Med. J., 29: 184-191.

Obeid, O.E., 2011. Characterization of human rotavirus subgroups and serotypes in children under five with acute gastroenteritis in a Saudi Hospital. J. Family Community Med., 18: 22-25.

Parashar, U.D., E.G. Hummelman, J.S. Bresee, M.A. Miller and R.I. Glass, 2003. Global illness and deaths caused by rotavirus disease in children. Emerg. Infect. Dis., 9: 565-572.

Ruiz-Palacios, G.M., I. Perez-Schael, F.R. Velazquez, H. Abate and T. Breuer et al., 2006. Safety and efficacy of an attenuated vaccine against severe rotavirus gastroenteritis. N. Engl. J. Med., 354: 11-22.

Settembre, E.C., 2011. Atomic model of an infectious rotavirus particle. EMBO J., 30: 408-416.

Steele, A.D., M. Patel, U.D. Parashar, J.C. Victor, T. Aguado and K.M. Neuzil, 2009. Rotavirus vaccines for infants in developing countries in Africa and Asia: Considerations from a world health organization sponsored consultation. J. Infect. Dis., 200: S63-S69.

Vesikari, T., D.O. Matson, P. Dennehy, P. van Damme and M. Santosham et al., 2006. Safety and efficacy of a pentavalent human-bovine (WC3) reassortant rotavirus vaccine. N. Engl. J. Med., 354: 23-33.

$\mathrm{WHO}, 2009$. Global and national estimates of deaths under age five attributable to rotavirus infection. World Health Organization, Geneva, Switzerland.

WHO, 2011. Estimated rotavirus deaths for children under 5 years of age: 2004, 527 000. Immunization Surveillance, Assessment and Monitoring, World Health Organization, Geneva, Switzerland.

Wang, F.T., T.C. Mast, R.J. Glass, J. Loughlin and J.D. Seeger, 2010. Effectiveness of the pentavalent rotavirus vaccine in preventing gastroenteritis in the United States. Pediatrics, 125: e208-e213.

Widdowson, M.A., D. Steele, J. Vojdani, J. Wecker and U. Parashar, 2009. Global rotavirus surveillance: Determining the need and measuring the impact of rotavirus vaccines. J. Infect. Dis., 200: S1-S8.

Zuccotti, G., F. Meneghin, D. Dilillo, L. Romano and R. Bottone et al., 2010. Epidemiological and clinical features of rotavirus among children younger than 5 years of age hospitalized with acute gastroenteritis in Northern Italy. BMC Infect. Dis., Vol. 10. 10.1186/1471-2334-10-218. 